\title{
CON LICENCIA PARA ARRIESGAR: UN MARCO TEÓRICO PARA LA ASIGNACIÓN DE RIESGOS EN ALIANZAS PÚBLICO - PRIVADAS BASADA EN LA ECONOMÍA DE COSTOS DE TRANSACCIÓN
}

\author{
LICENSED TO RISK: A THEORETICAL FRAMEWORK FOR ASSIGNING RISK ON \\ PUBLIC PRIVATE PARNERTSHIPS BASED ON TRANSACTION COST ECONOMICS
}

\author{
Po Chun Lee \\ po.lee@iaen.edu.ec
}

\section{Resumen:}

Este artículo empieza explorando el significado de las alianzas o asociaciones público-privadas (APP) apuntando a múltiples interpretaciones que revisan la literatura sobre las APP. El documento identifica que uno de los grandes problemas en las APP es que carecen de soporte teórico, y en la práctica se viola el derecho al reclamo. Por lo tanto, es necesario contar con mecanismos formales para interpretar por qué un gobierno retiene un riesgo particular en un proyecto mientras se transfiere recursos a socios privados. Desde el punto de vista de la economía de costos de transacción (ECT), integrada con la visión basada en recursos de las capacidades organizacionales (RCO), este documento propone un marco teórico para entender la práctica de asignación de riesgos en proyectos de APP. Este marco teórico proporciona a las agencias gubernamentales y privadas una comprensión lógica y completa del proceso de selección de la estrategia de asignación para un riesgo particular en los proyectos de APP. Además, podría utilizarse para dirigir la estrategia de asignación de riesgos controlando ciertos determinantes críticos identificados en el estudio. El trabajo también establece sus limitaciones y las futuras direcciones de investigación.

Palabras claves: alianza público- privada; economía de costos de transacción; metodología asignación de riesgos

\section{Abstract:}

This article starts by exploring the meaning of public private partnerships (PPP), aiming at the multiple interpretations in the APP literature. This document identifies that some of the issues regarding APP's failure are due to the lack of technical support and when faced with hardships, parties tend to violate the right to appeal the process. Therefore, it is necessary to conceive formal mechanisms for interpreting the inherent risk of transferring resources from the government to the private party. From of the point of view transaction cost economics (TCE), and the Resource Based View (RBV) of organizational capacity, this paper proposes a theoretical framework to understand the risk management for PPP. This framework allows government agencies and private parties to have a logic understanding for the process of APP project procurement and the allocation of risk. This work also establishes its limitations and the future direction of its research.

Keywords: public private partnerships, transaction cost economics; risk assignment methodology.

\section{INTRODUCCIÓN}

El rápido crecimiento poblacional y súbita urbanización en muchos países ha llevado a una demanda masiva de inversión en infraestructura (Yehoue y Ruhashyankio, 2006). La provisión convencional de infraestructura financiada por los gobiernos ha conducido a ineficiencias y ha sometido el desarrollo de la infraestructura a la 
disponibilidad de fondos gubernamentales. Como mecanismo para equilibrar tales anomalías, una variedad de acuerdos de alianza público-privada (APP) se está convirtiendo rápidamente en la forma preferida de proporcionar servicios públicos en muchos países (Doloi y Jin, 2008). La APP se ha interpretado ampliamente para abarcar cualquier acuerdo entre el gobierno y el sector privado para prestar servicios al público (Klijn y Teisman, 2002). Sin embargo, las APP, en este estudio, se refieren a un acuerdo contractual complejo a largo plazo que involucra la prestación de servicios que requieren la construcción de activos de infraestructura. Aunque hay muchas partes involucradas en una transacción APP, el enfoque en este estudio se centra en dos amplios grupos de partes interesadas, es decir, la agencia gubernamental y el consorcio privado, entre quienes se asignan los riesgos (Grimsey y Lewis, 2007).

El principio central para las APP es la relación calidad-precio (RCC). Uno de los principales factores de RCC es la transferencia de riesgos, lo que significa que los riesgos apropiados se pueden transferir al sector privado, que se supone que es capaz de gestionar mejor esos riesgos (Kwak et al., 2009). En consecuencia, los servicios de infraestructura pueden proporcionarse de manera más económica y de mayor calidad que en la forma convencional. Sin embargo, la complejidad de los arreglos y la naturaleza de contratación incompleta han llevado a una mayor exposición al riesgo para todas las partes involucradas (Shen y Deng, 2006). La asignación efectiva de riesgos en los proyectos de APP no es, por lo tanto, un trabajo fácil. La asignación de riesgos por una agencia gubernamental y la compartición de este con el socio privado requiere de mecanismos que orienten la formación de estrategias de asignación de riesgos. La respuesta a este desafío es críticamente importante para el éxito o fracaso de los proyectos de APP (Jin y Doloi, 2007).
El argumento principal de este trabajo radica en sugerir un marco teórico para abordar la cuestión desde la perspectiva de la economía de costos de transacción (ECT), integrada con la perspectiva basada en los recursos de las capacidades de la organización (RCO). Esto es parte de un gran proyecto que apunta al desarrollo de técnicas sólidas basadas en el conocimiento del sistema, capaces de mejorar la asignación de riesgos y los procesos de gestión.

En la siguiente sección, se aclaran las definiciones y dimensiones de las APP. El documento continúa con la construcción del marco teórico, introduciendo los conceptos necesarios para construir la metodología y discutiendo las prácticas de asignación de riesgos en los proyectos de APP, la capacidad de organización y los costos de transacción. El marco teórico y sus constructos claves se describen a continuación, seguida de una discusión sobre las limitaciones de la investigación y las direcciones futuras. Finalmente, se presenta una breve conclusión.

\section{Definiciones y dimensiones de las Alianzas Público-Privadas}

Las APP han visto una variedad de definiciones. Garvin y Bosso (2008), por ejemplo, definieron los APP como "un acuerdo contractual a largo plazo entre los sectores público y privado donde se buscan beneficios mutuos donde el sector privado proporciona servicios de gestión y operación y pone en riesgo la financiación privada." La Organización para la Cooperación y el Desarrollo Económico definió las APP como un acuerdo entre el gobierno y uno o más socios privados. Este acuerdo puede incluir a los operadores y los financiadores que entregan un servicio que este alineado a los objetivos de eficacia y transferencia de riesgo de las partes involucradas (OCDE, 2008). Otros han visto APP más ampliamente, Osei-Kyei y Chan (2015) lo perciben como una "cooperación entre actores público-privados en la que desarrollan productos y servicios en forma 
conjunta y comparten riesgos, costos y recursos". Pessoa (2008) argumentó que, como casi todos los proyectos de infraestructura pública involucran a los sectores público y privado de una forma u otra, "todos los proyectos son, por lo tanto, una asociación público-privada". Y más ampliamente, autores como Hodge y Greve (2009) han colocado las APP de infraestructura como una de varias familias diferentes de actividades de asociación entre los dos sectores.

Aquí hay varios conceptos cruciales. Un concepto es el "riesgo". En casi todas las definiciones, el compartir los riesgos de una manera explícita se menciona como uno de los aspectos clave de APP (Grimsey y Lewis, 2002). Esto difiere de las ideas anteriores sobre el riesgo compartido a través de acuerdos de subcontratación (Kwak, Chih y Ibbs, 2009). Otro concepto clave es la "innovación" donde el sector público y el sector privado tienen que encontrar nuevas soluciones y trabajar juntos para lograr un objetivo común (Shou y Chen, 2017). También hay una expectativa de que la relación sea a largo plazo y más larga que una relación tradicional de servicios o contratación externa (Shou y Chen, 2017).

Además, la noción de APP mantiene cierto grado de poder compartido mientras laboren juntas. La APP con contrato de infraestructura a largo plazo (CILP) ahora es prominente. La APP de CILP se organiza típicamente en torno a un modelo de diseño, finanzas, construcción, propiedad, operación, transferencia e involucra el financiamiento del sector privado y las capacidades de gestión de proyectos del sector privado (Shen, Platten y Deng, 2006).

Aunque el debate sobre las APP puede ser complejo en las disciplinas de ingeniería y finanzas de proyectos, siguen siendo obstinadamente ambiguas, fluidas y resbaladizas en las disciplinas de la ciencia política y la administración y gestión pública. Tal vez el problema más importante aquí es la necesidad de pensar en APP no simplemente como un acuerdo de entrega de proyectos, sino de una manera más sofisticada; como un fenómeno que cubre un debate de cinco dimensiones:

1. Un proyecto o actividad de infraestructura específica;

2. Una forma de organización, acuerdo de entrega de proyecto o una herramienta de gestión; 3. Una política, declaración o símbolo en cuanto al papel del sector privado en una economía mixta;

4. Una herramienta o estilo en la tarea de gobierno moderno; y

5. Un fenómeno dentro del contexto de una historia nacional más amplia entrañado con un conjunto de suposiciones culturales.

En el nivel más estrecho de la dimensión, una APP se lo puede ver como un proyecto único. Añadiendo una capa de complejidad, una APP puede verse como un tipo de mecanismo específico para la entrega de infraestructura con una arquitectura institucional $y$ financiera específica. Si bien las concepciones de APP en las últimas dos décadas han cubierto el financiamiento tanto público como privado, la preferencia más reciente ha visto cada vez más que esta arquitectura de entrega asume la financiación privada, lo cual, se argumenta, fomenta el rendimiento superior del proyecto $\mathrm{y}$ la entrega temprana (Osei-Kyei y Chan, 2015).

El próximo nivel conceptual lleva esta herramienta de proyecto un paso más allá y establece la entrega de financiamiento privado de la infraestructura como una preferencia de política para una jurisdicción. De hecho, en algunas jurisdicciones, puede convertirse en la única opción para la realización de grandes infraestructuras (Pessoa, 2008). También puede operar a un nivel de política más amplio no como un solo tipo de APP, sino que reconoce una amplia variedad de opciones alternativas de entrega de proyectos disponibles para los gobiernos. La lista de opciones APP presentada por Little (2011) o la lista de siglas de la OCDE son manifestaciones de esto. La amplitud 
disponible aquí es, por lo tanto, esencialmente una declaración de política según la cual el sector privado tiene un papel válido y de hecho importante en la economía mixta actual, sea cual sea la opción de entrega técnica que se elija.

Ampliando nuevamente en este modelo conceptual, la cuarta dimensión representa el grado en que las APP siempre han tenido una dimensión de gobernanza inherente. Para empezar, el uso de grandes consorcios privados para la entrega de proyectos gubernamentales de alto perfil es una herramienta reguladora sólida en el gobierno, porque no solo da forma a la arquitectura de opciones disponibles al público, sino que también se pueden emplear grandes incentivos económicos para asegurar que se cumpla la promesa los objetivos gubernamentales anticipadamente. Las APP, en este caso, pueden funcionar como una herramienta de gobernanza más amplia y marcar un estilo particular de gobierno.

La quinta dimensión conceptual tiene que ver la implicación del uso de metodologías de gestión de proyectos que son cada vez más profesionales y puestas a prueba a través de nuevos acuerdos comerciales, financieros $\mathrm{e}$ institucionales, en contraste a la preferencia de los métodos tradicionales de la burocracia pública. En este sentido, la quinta dimensión de APP representa un movimiento simbólico importante para innovar, comercializar y profesionalizar. En la quinta dimensión de este modelo conceptual, la marca APP representa el cambio cultural en la gestión pública-privada.

\section{Conceptos para la asignación de riesgos en proyectos de APP}

El riesgo es "la posibilidad de que algo suceda que tendrá un impacto sobre los objetivos"(Hans et al., 2004). Los proyectos de construcción manifiestan más riesgos que otras industrias (Hans et al., 2004). El éxito de un ejercicio de gestión de proyectos depende en gran medida en que los riesgos involucrados puedan ser identificados, medidos, comprendidos, informados, comunicados y asignados a las partes apropiadas (Hodge y Greve, 2009). Sin embargo, la evidencia de proyectos en todo el mundo muestra que este no es un evento directo y que los riesgos no se manejan adecuadamente (Kwak y Ibbs, 2009).

En los proyectos de APP, es fundamental que el gobierno entienda que no es óptimo para ellos no solo retener sino también transferir riesgos inapropiados. Esto se debe a que la transferencia de riesgos al sector privado tiene un precio y la asignación inadecuada de riesgos entre las partes interesadas puede generar precios superiores a los necesarios (Thomas et al., 2003). La asignación inadecuada de riesgos también puede dañar la Relación Costo Calidad (RCC) porque las medidas del costo total del proyecto son muy sensibles a la asignación de riesgos. Si los riesgos son inapropiados para el sector público, el gobierno aumentaría los impuestos o reduciría los servicios para pagar sus obligaciones cuando se materialicen los riesgos. Por el contrario, si los riesgos son inadecuados para el sector privado, las primas excedentes se cobrarían al gobierno o incluso directamente a los usuarios finales (Thomas et al., 2003).

Muchos gobiernos ahora reconocen que la privatización es una asociación en la que deben mantener cierto riesgo. Algunos proyectos pueden ser más difíciles de implementar y aplicar, política o socialmente, que otros, particularmente si existe una fuerza de trabajo del sector público que teme ser transferida al sector privado o si existen cambios significativos como pugnas sobre tierras o reasentamientos. En estos casos, se requieren aumentos significativos en las tarifas para que el proyecto sea viable. No solo eso, la responsabilidad del gobierno continúa aún después de ejecutado el proyecto: los ciudadanos pueden continuar responsabilizando al gobierno por la calidad de los servicios públicos. Por ende, el gobierno deberá implementar una agencia de ejecución o un organismo regulador para poder cumplir sus 
propias obligaciones bajo el acuerdo de APP y monitorear el desempeño y cumplimiento del sector privado (Grimsey y Lewis, 2002).

Es más, dada la naturaleza a largo plazo de estos proyectos y la complejidad asociada, es difícil identificar todas las contingencias posibles durante el desarrollo del proyecto $\mathrm{y}$ pueden surgir eventos y problemas que no fueron anticipados en los documentos o por las partes en el momento del contrato (Grimsey y Lewis, 2002). Es más probable que las partes necesiten renegociar el contrato para acomodar estas contingencias. También es posible que algunos proyectos fallen o se rescindan antes del plazo proyectado, o por varios motivos, incluidos cambios en la política gubernamental, incumplimiento por parte del operador privado o del gobierno en el cumplimiento de sus obligaciones o, de hecho, debido a circunstancias externas como fuerza mayor (Pongsiri, 2002).

Sin embargo, la percepción de que la privatización implica la transferencia de todos los riesgos al sector privado aún prevalecía en muchos países hasta hace poco (Faulkner, 2004). En ocasiones, los riesgos se asignarán inevitablemente a la parte menos capaz de rechazarlos en lugar de a la parte mejor capacitada para administrarlos, especialmente cuando el gobierno mantiene la tensión competitiva máxima (Thomas et al., 2003). Se ha dejado expresamente en claro a la parte privada que la asignación se ofrece para su aceptación y se espera poca divergencia (Thomas et al., 2003). Esta estructura crea implícitamente una asignación de riesgo inicial en la que todos los riesgos asociados con la entrega de los resultados a los estándares de servicio especificados se asignan a la parte privada.

No obstante, se han realizado esfuerzos constantes para buscar una asignación de riesgo óptima investigando qué categorías de riesgo los gobiernos deberían aceptar o transferir en general y, críticamente, por qué. La asignación óptima de riesgos busca minimizar los riesgos para el proyecto asignando riesgos particulares a la parte que se encuentre en la mejor posición para controlarlos (Faulkner, 2004). Esto se debe a que la parte que posee la mejor capacidad de gestión con respecto a un riesgo particular tiene la mejor oportunidad de reducir la probabilidad de que se produzca el riesgo y controlar las consecuencias del riesgo si se materializa, y si así debería asumirlo (Thomas et al., 2003).

\section{Capacidades organizacionales}

Dado que las capacidades de Gestión de Riesgo (GR) de las partes involucradas se han convertido en la mayor preocupación cuando se elige una estrategia de asignación de riesgos, es necesaria una revisión de la capacidad de la organización. La noción de capacidades se remonta al trabajo de Penrose (1959). Si bien los recursos están disponibles para todas las empresas, la 'capacidad' para desplegarlos productivamente no está distribuida uniformemente (Penrose, 1959). Con la evolución de la visión basada en recursos (VBR), se ha reconocido cada vez más que VBR explica la heterogeneidad competitiva basada en la premisa de que los competidores cercanos difieren en sus capacidades y recursos de maneras importantes y duraderas (Kor y Mahoney 2004). La literatura sobre VBR conceptualiza recursos y capacidades de dos maneras diferentes. Barney, Wright y Ketchen (2001) tendían a definir los recursos de manera amplia al incluir todos los activos, capacidades, procesos organizacionales, atributos de la empresa, información, conocimiento.

Sin embargo, otro conjunto de autores (véase, por ejemplo, Wu, 2010; Lin \& Wu, 2014; Shou, Shao \& Chen, 2017) buscaron delinear claramente entre recursos y capacidades argumentando que los recursos son accesibles para todas las empresas a precios de factor prevalecientes, mientras que las capacidades se refieren a la capacidad de una empresa para desplegar recursos. En este documento, se adoptó la última definición de capacidades organizativas. 


\section{Economía de costo de transacción (ECT)}

La asignación de riesgos en los proyectos de APP es adecuada para ser vista desde la perspectiva de ECT, ya que cualquier problema que pueda formularse como un problema de contratación puede investigarse con ventaja en términos de economía de costos de transacción (Williamson, 1985). La idoneidad también surge de muchas características de las APP, que incluyen contratos incompletos, asociaciones a largo plazo, fuertes inversiones en activos, incertidumbre compleja, etc. (Jin y Doloi, 2007).

Los costos de transacción son los costos de funcionamiento del sistema económico. Dichos costos son el equivalente económico de la fricción en los sistemas físicos y se distinguen de los costos de producción (Williamson, 1985). ECT plantea el problema de la organización económica como un problema de contratación y supone que los agentes humanos están sujetos a una racionalidad limitada, donde el comportamiento es (1) "intencionalmente racional, pero solo de forma limitada" y (2) dado al oportunismo, que es una condición del "interés propio que busca con astucia" (Williamson, 1985).

ECT también sostiene que existen razones económicas racionales para organizar algunas transacciones de una manera y otras transacciones de otra. Las principales dimensiones con respecto a las cuales las transacciones difieren son (1) la especificidad de los activos, el grado en que un activo puede ser redistribuido a usos alternativos y por usuarios alternativos sin sacrificar el valor productivo (Williamson, 1985); (2) incertidumbre, que puede surgir del "estado de la naturaleza" o cambios en el ambiente externo que afectan un sistema o cuando se unen la contratación incompleta y la especificidad de los activos (Williamson, 1996); y (3) frecuencia que admite el hecho de que la identidad por pares de las partes importa y tiene consecuencias generalizadas para la organización de la actividad económica.
El imperativo organizacional consiguiente es "organizar las transacciones para economizar en la racionalidad limitada al tiempo que las protege contra los riesgos del oportunismo". Al asignar las transacciones a las estructuras de gobierno de una manera discriminatoria, los costos de transacción se economizan (Williamson, 1985). Sin embargo, los costos de transacción siempre se evalúan de una manera institucional comparativa (Williamson, 1996). La investigación empírica sobre los problemas del costo de transacción permanece si las relaciones organizacionales se alinean con los atributos de las transacciones como lo predice el razonamiento del costo de la transacción o no (Williamson, 1985). Aunque el VBR de la capacidad organizativa y el ECT están estrechamente vinculados con el análisis de la asignación de riesgos, se ha realizado poca investigación al integrar estas dos teorías. Esto podría deberse a la larga discusión existente entre ellos.

La literatura sobre ECT, por ejemplo, sostiene que gran parte de la literatura sobre capacidades organizacionales no presta atención al entorno comercial y el potencial resultante para el oportunismo (Mahoney, 2001). Por otro lado, la literatura sobre capacidades organizacionales sostiene que ECT subestima las diferencias en las capacidades de la empresa. Se presenta en este documento, sin embargo, que el VBR de la capacidad de organización y ECT son de naturaleza complementaria. Esto se debe a que el papel de la especificidad de los activos, que es uno de los componentes más críticos en ECT, es más evidente en la descripción de la capacidad organizacional (Barney, Wright y Ketchen, 2001). Además, si bien ECT sostiene que la frecuencia de las transacciones es importante, el VBR de la capacidad organizacional atiende al hecho de que las transacciones pasadas entre socios pueden generar procesos que alteran el cálculo para transacciones futuras (Rao, 2003). Esto hace que las formulaciones de ECT y VBR de la capacidad organizacional se superpongan. 


\section{METODOLOGÍA}

Si bien la capacidad organizacional en la Gestión de Riesgos (GR) sigue siendo una de las principales preocupaciones, se presenta en este documento que la toma de decisiones sobre la asignación de riesgos se puede explicar mejor cuando se adopta una visión holística. Esto podría lograrse integrando el concepto de Economía de Costo de Transacción (ECT) con una Visión Basada en Recursos (VBR) de capacidad organizativa, teniendo en cuenta otros factores importantes, como las incertidumbres. Por lo tanto, la elección de una estrategia de asignación de riesgos podría considerarse como el proceso de decidir la proporción de responsabilidad de gestión de riesgos entre las organizaciones internas y externas en función de una serie de características de la transacción de servicio de GR en cuestión. Las características se pueden categorizar en (1) la capacidad de GR de los socios, que es la capacidad de organización en la VBR y puede considerarse como los principales activos especificados de ECT; (2) el historial de cooperación de los socios, que se aproxima a la frecuencia de las transacciones de ECT; (3) incertidumbre del entorno de GR; y (4) el compromiso de GR de los socios, que coincide con la incertidumbre conductual de ECT. En consecuencia, un marco teórico como el que se muestra en la Figura 1 se establece sobre la base de las siguientes hipótesis principales:

Hipótesis 1: capacidad de GR de los socios (activos específicos), historial de cooperación de los socios (frecuencia de transacción) e incertidumbres del entorno determinan el compromiso de GR de los socios.

Hipótesis 2: la capacidad de GR de los socios (activos específicos), el historial de cooperación de los socios (frecuencia de transacción), las incertidumbres del entorno y el compromiso de GR de los socios (incertidumbres de comportamiento) determinan la estrategia de asignación de riesgos (estructura de gobierno).

Los constructos y sus enlaces se discuten a continuación y se lo consolida en un marco metodológico en la Figura 1.

\section{Figura 1 - Marco Teórico para la Gestión de Riesgos en proyectos de Alianza Público Privada}

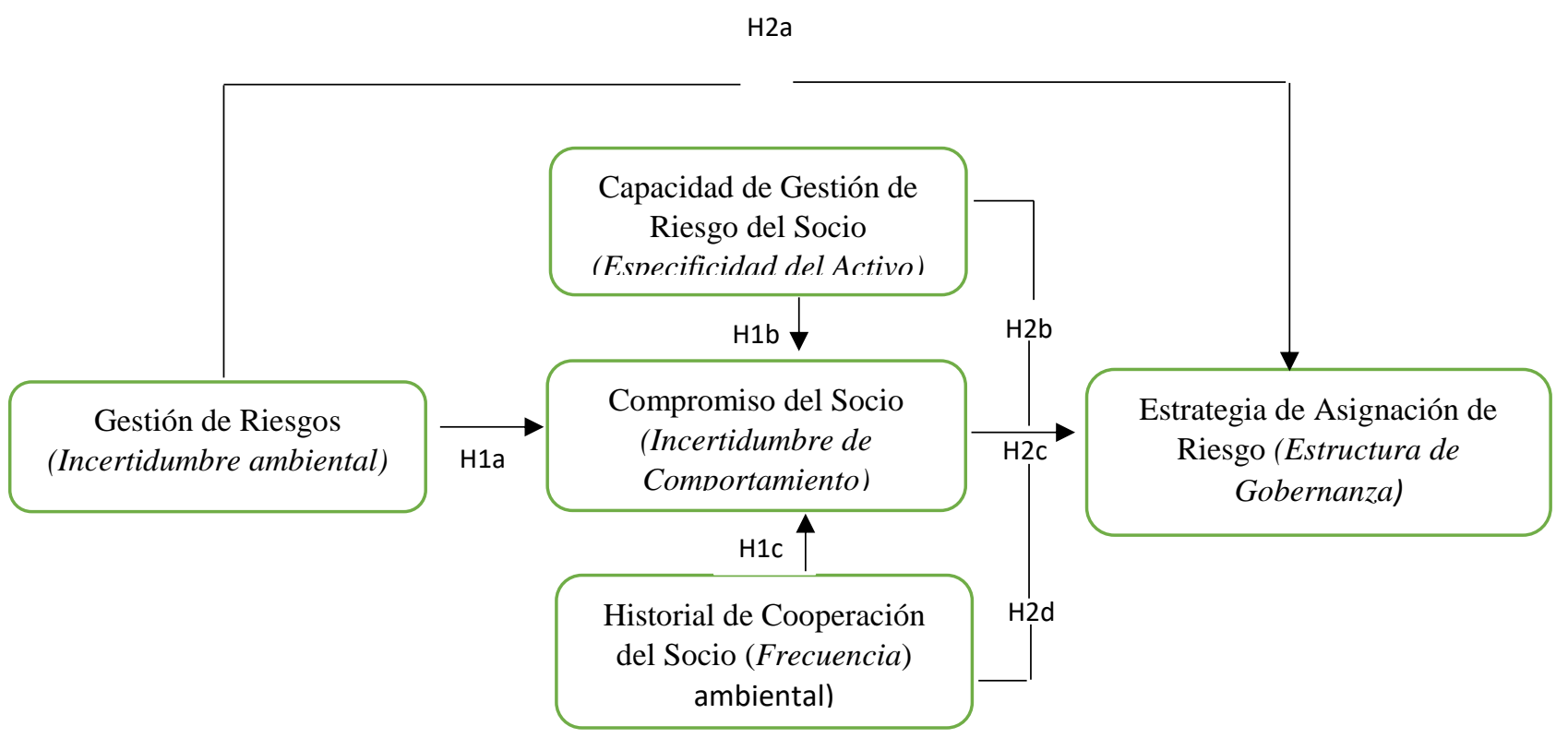




\section{RESULTADOS}

\section{Capacidad organizativa (activos específicos)}

En este estudio se propone que los activos específicos más importantes en cualquier transacción de servicio de Gestión de Riesgos serían la capacidad de Gestión de Riesgo Organizacional. Esto se debe a que el papel de la especificidad de los activos es más evidente en la descripción de la capacidad de la organización y, por lo tanto, se mantiene más cerca de las formulaciones de ECT (Rao, 2003). La capacidad de la Gestión de Riesgos puede ser operacionalizada en la rutina y el mecanismo de RG.

\section{Rutina de Gestión de Riegos (especificidad de activos)}

Las organizaciones desarrollan capacidades llevando a cabo actividades relacionadas repetidamente. Las diferencias en actividades pasadas conducen a capacidades heterogéneas. Las empresas son entidades que poseen capacidades heterogéneas en función de sus rutinas y procesos de búsqueda (Nelson y Winter, 1982). Con el tiempo, el conocimiento acumulado a través de 'aprender haciendo' está integrado en paquetes de 'rutinas' que se asemejan al material genético de la empresa y sirven como memoria organizacional para ejecutar repetitivamente la secuencia de actividades productivas.

Aunque las rutinas representan el conocimiento y la competencia para llevar a cabo actividades de gestión de riesgo. Estas rutinas también indican que se podrían lograr usos alternativos sin sacrificar el valor productivo. Es decir, cuanto más larga es la historia de una rutina de GR, más uso alternativo del activo se acumula, y por lo tanto menos especifico se convierte. Esto es particularmente comprensible en el contexto de la industria de la construcción, donde los productos son generalmente de naturaleza única, a pesar de que la rutina sea repetitiva. Esto se distingue de la naturaleza repetitiva en industrias tales como la manufactura (donde la rutina es repetitiva y el producto es uniforme).

\section{Mecanismo de Gestión de Riesgo (vencimiento de activos específicos)}

Las capacidades tienden a evolucionar a lo largo del tiempo para reflejar los efectos conjuntos del aprendizaje pasivo y las inversiones deliberadas a nivel de empresa para aprender y hacer mejoras (Kor y Mahoney, 2004). Las capacidades tienen más probabilidades de desarrollarse eficazmente cuando se establecen mecanismos diseñados para acumular, almacenar, integrar y difundir el conocimiento organizacional relevante adquirido a través de la experiencia (Klijn, E. H. y Teisman). Estos mecanismos integradores actúan como un lugar importante de aprendizaje firme. Por lo tanto, aunque una mayor experiencia en GR puede ser una condición necesaria para que las organizaciones creen capacidad de GR, esto no es necesariamente condicionante. La capacidad de GR también depende de cuán efectivamente es capaz la organización de capturar, compartir y diseminar el mecanismo de gestión de riesgo.

\section{Estrategias de asignación de riesgos (estructura de gobernanza)}

La asignación de riesgos es el proceso de dividir y asignar la responsabilidad asociada con un riesgo particular para una variedad de circunstancias hipotéticas (Uff, 1995). Se argumentó como una transacción de responsabilidad de gestión de riesgos entre los posibles portadores de riesgos. Por lo tanto, las diferentes estrategias de asignación son, de hecho, diferentes estructuras de gobernanza de la gestión del riesgo. La Economía de Costos de Transacción sostiene que, con una cierta combinación de las dimensiones de transacción, una organización responde a ellas adoptando una estructura de gobierno para economizar en los costos de transacción (Williamson, 1985). 
Las estructuras incluyen la jerarquía interna, la de mercado y un modo híbrido (Williamson, 1996). En consecuencia, en una situación dada de las características antes mencionadas en un proyecto de APP, los socios acordarán una estrategia de asignación de riesgo específica, es decir, una proporción específica de un riesgo dado para ser transferido de un socio público a un socio privado para reducir los costos de transacción. Esta proporción o estrategia puede ser del $100 \%$ (transferencia total o pagar por el proyecto), $0 \%$ (retener por completo y hacer el proyecto), o en algún punto intermedio (hacer y pagar por el proyecto proporcionalmente; eg. 5050). Este trabajo, en cambio, se interesa en las transacciones donde se puede proporcionar la transferencia de riesgos basados en el concepto de ECT de economizar costos de transacción, y el mecanismo inherente que asigna a diferentes estructuras de gobierno la gestión de riesgos.

\section{Limitación e investigación futura}

Una limitación importante en este estudio es que se consideró una relación lineal solo entre las variables explicativas y de respuesta. Sin embargo, la no linealidad no es poco común en situaciones realistas. Otra limitación es que los métodos lineales están básicamente orientados a la probabilidad e incapacidad de identificar todos los factores necesarios para reflejar situaciones realistas (Thomas et al., 2003).

El mecanismo de gestión de riesgos de los socios, por ejemplo, no se incluyó en el modelo óptimo para la estrategia de asignación de riesgos, aunque teóricamente tiene una relación causal con la transferencia de riesgos.

Un modelo que se puede aplicar a la metodología, una vez que se obtengan los datos, es usando las redes neuronales artificiales ya que ayudan a que el sistema sea adecuado para la predicción. Esta técnica se ha adoptado en las etapas posteriores de la investigación actual, que se informará en otro artículo. Como se mencionó anteriormente, aunque el marco propuesto puede facilitar la comprensión del mecanismo actual de asignación de riesgos, no puede decir si la práctica actual es óptima o no. En las etapas posteriores del estudio actual, se construyó un sistema de inferencia difusa basado en el conocimiento para identificar estrategias de asignación de riesgos óptimas y subóptimas. Se construyó un flujograma para predecir la estrategia óptima de asignación de riesgos en diferentes escenarios de proyectos. Por lo que las aplicaciones de las técnicas de inteligencia artificial se informarán en artículos separados.

\section{Conclusión}

Este artículo empezó investigando el significado de las alianzas o asociaciones público-privadas (APP) señalando las variadas elucidaciones que revisan la literatura sobre las APP. Para esto se consideró las cinco dimensiones de las APP que van desde la concepción de un proyecto o actividad de infraestructura específica, así como una herramienta de gestión, o de un símbolo político para demostrar la cooperación entre el sector público y privado que conformaría una de las tareas del gobierno moderno; $\mathrm{y}$, por último, un fenómeno con dimensiones y un conjunto de suposiciones culturales.

La complejidad de las APP y sus dimensiones se plasmó con el concepto de riesgo. Este trabajo trató de precisar el riesgo según el concepto de la Economía de Costos de Transacción (ECT), que está ligada intrínsecamente con la especificidad de los activos, la incertidumbre que puede surgir de los cambios en la contratación incompleta y la frecuencia de las transacciones entre las partes. Integrando ECT con la perspectiva basada en los recursos de las capacidades organizativas (RCO), este trabajo parte de un gran proyecto que apunta al desarrollo de técnicas basadas en el conocimiento del sistema, capaces de mejorar la asignación de riesgos y los procesos de gestión. 
Fundamentalmente, este artículo propuso un marco teórico para comprender el mecanismo subyacente de la toma de decisiones de asignación de riesgos en los proyectos de APP desde la perspectiva de ECT. Al integrar aún más la visión basada en los recursos de las capacidades organizativas, este marco permite una interpretación lógica y holística del mecanismo subyacente al proceso de toma de decisiones en la práctica actual de asignación de riesgos. Los principales componentes incluyen la rutina de gestión de riesgos de los socios, el mecanismo, el historial de cooperación, el compromiso y la incertidumbre del entorno.

Sus enlaces se presentaron como dos hipótesis principales, a saber: (1) la capacidad de GR de los socios, el historial de cooperación de los socios y las incertidumbres ambientales determinan el compromiso de GR de los socios; y (2) la capacidad de GR de los socios, el historial de cooperación de los socios, las incertidumbres ambientales y el compromiso de GR de los socios determinan la estrategia de asignación de riesgos.

\section{REFERENCIAS BIBLIOGRÁFICAS}

Barney, J., Wright, M. y Ketchen Jr, D. J. (2001). The resource-based view of the firm: Ten years after 1991. Journal of management, 27(6), 625-641.

Faulkner, K. (2004). Public-private partnerships. Public-private partnerships: Policy and experience, New York: Palgrave Macmillan, 6570.

Garvin, M. J. y Bosso, D. (2008). Assessing the effectiveness of infrastructure public - private partnership programs and projects. Public Works Management \& Policy, 13(2), 162-178.
Es merecido mencionar que el historial de cooperación de los socios (es decir, la frecuencia de las transacciones en ECT) también pueden tener efectos indirectos en la toma de decisiones sobre la asignación de riesgos al conformar el compromiso de los socios. Además, el compromiso de GR de los socios puede verse significativamente manipulado por la situación de varios factores ambientales y empresariales.

Con el marco basado en ECT, la toma de decisiones sobre la asignación de riesgos fue mejor interpretada. En el caso de grandes inversiones específicas de GR, podría ser más eficiente para el socio público retener la mayoría o la totalidad del riesgo dado. Sin el compromiso apropiado del socio privado es probable que no logren administrar ese riesgo de manera eficiente mediante el cobro de primas más altas, incluso con una excelente capacidad de GR. Del mismo modo, un entorno comercial volátil debe tomarse como una señal de advertencia, donde el socio público no debe transferir tanto riesgo como lo desee. Si lo hacen bajo una mayor incertidumbre, el proyecto puede terminar con un costo superior al necesario.

Grimsey, D. y Lewis, M. K. (2002). Evaluating the risks of public private partnerships for infrastructure projects. International journal of project management, 20(2), 107-118.

Grimsey, D. y Lewis, M. (2007). Public private partnerships: The worldwide revolution in infrastructure provision and project finance. Edward Elgar Publishing.

Han, S. H., Diekmann, J. E., Lee, Y. y Ock, J. H. (2004). Multicriteria financial portfolio risk management for international projects. Journal of construction engineering and management, 130(3), 346-356. 
Hodge, G. A. y Greve, C. (2009). PPPs: The passage of time permits a sober reflection. Economic Affairs, 29(1), 33-39.

Jin, X. y Doloi, H. (2007, January). Risk allocation in public-private partnership projects: an innovative model with an intelligent approach. In COBRA 2007: Proceedings of the Construction and Building Research Conference of the Royal Institution of Chartered Surveyors (pp. 1-13). Royal Institution of Chartered Surveyors (RICS).

Jin, X. H. y Doloi, H. (2008). Interpreting risk allocation mechanism in public-private partnership projects: an empirical study in a transaction cost economics perspective. Construction Management and Economics, 26(7), 707-721.

Klijn, E. H. y Teisman, G. R. (2002). Governing public-private partnerships: Analysing and managing the processes and institutional characteristics of public-private partnerships. In Public-private partnerships (pp. 102-120). Routledge.

Kor, Y. Y. y Mahoney, J. T. (2004). Edith Penrose's (1959) contributions to the resourcebased view of strategic management. Journal of management studies, 41(1), 183-191.

Kwak, Y. H., Chih, Y. y Ibbs, C. W. (2009). Towards a comprehensive understanding of public private partnerships for infrastructure development. California management review, 51(2), 51-78.

Lin, Y. y Wu, L. Y. (2014). Exploring the role of dynamic capabilities in firm performance under the resource-based view framework. Journal of business research, 67(3), 407-413.
Little, R. G. (2011). The emerging role of publicprivate partnerships in megaproject delivery. Public Works Management \& Policy, 16(3), 240-249.

Mahoney, J. T. (2001). A resource-based theory of sustainable rents. Journal of management, 27(6), 651-660.

Nelson, R. y Winter, S. (1982). An evolutionary theory of the firm. Belknap, Harvard, 41.

Osei-Kyei, R. y Chan, A. P. (2015). Review of studies on the Critical Success Factors for Public-Private Partnership (PPP) projects from 1990 to 2013. International Journal of Project Management, 33(6), 1335-1346.

Penrose, E. T. (1959). The theory of the growth ofthe firm. New York: Sharpe

Pessoa, A. (2008). Public-private partnerships in developing countries: are infrastructures responding to the new ODA strategy?. Journal of International Development, 20(3), 311-325.

Pongsiri, N. (2002). Regulation and publicprivate partnerships. International Journal of Public Sector Management, 15(6), 487-495.

Rao, P. (2002). The Economics of Transaction Costs: Theory, Methods and Application. Springer.

Shen, L. Y., Platten, A. y Deng, X. P. (2006). Role of public private partnerships to manage risks in public sector projects in Hong Kong. International journal of Project management, 24(7), 587-594. 
Shou, Y., Shao, J., y Chen, A. (2017). Relational resources and performance of Chinese thirdparty logistics providers: The mediating role of innovation capability. International Journal of Physical Distribution \& Logistics Management, 47(9), 864-883.

Thomas, A. V., Kalidindi, S. N. y Ananthanarayanan, K. A. B. T. (2003). Risk perception analysis of BOT road project participants in India. Construction Management and Economics, 21(4), 393-407.

Williamson, O.E. (1985) The Economic Institutions of Capitalism: Firms, Markets, Relational Contracting, Free Press, New York.

Williamson, O. E. (1996). The mechanisms of governance. Oxford University Press.

Wang, S. Q., Dulaimi, M. F. y Aguria, M. Y. (2004). Risk management framework for construction projects in developing countries. Construction Management and Economics, 22(3), 237-252.

Wu, L. Y. (2010). Applicability of the resourcebased and dynamic-capability views under environmental volatility. Journal of Business Research, 63(1), 27-31.

Yehoue, M. E. B., Hammami, M. y Ruhashyankiko, J. F. (2006). Determinants of public-private partnerships in infrastructure (No. 6-99). International Monetary Fund. $\mathrm{h}$ ttps://www.imf.org/external/pubs/ft/wp/2006/w p0699.pdf 